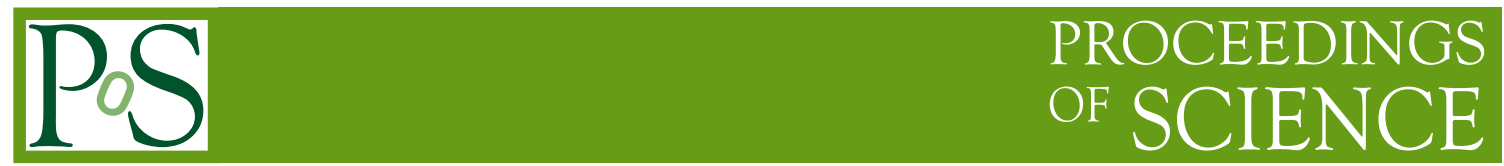

\title{
Dark matter from evaporating Black Holes
}

\section{Isabella Masina ${ }^{a, b, *}$}

${ }^{a}$ University of Ferrara, Dept. of Physics and Earth Sciences

Via Saragat 1, 44100 Ferrara, Italy

${ }^{b}$ INFN, Sez. Ferrara,

Via Saragat 1, 44100 Ferrara, Italy

E-mail: masina@fe.infn.it

Light primordial black holes (PBHs) are an ideal particle factory: they emit all existing particles - of the Standard Model (SM) or beyond it - whose mass is smaller than the PBHs Hawking temperature. Evaporation of PBHs is thus an interesting mechanism for Dark Matter (DM) production. We review the status of the art of this possibility, analyzing in particular the effects of the DM particle spin, for both Schwarzschild and Kerr PBHs.

*** The European Physical Society Conference on High Energy Physics (EPS-HEP2021), ***

*** 26-30 July $2021 * * *$

*** Online conference, jointly organized by Universität Hamburg and the research center DESY ***

${ }^{*}$ Speaker 


\section{Introduction on PBHs formation and evaporation}

PBHs could have formed in the very early Universe, during the radiation dominated era at the end of inflation, due to gravitational collapse of over-dense regions. There are several mechanisms for PBHs formation - see e.g. the review by Carr et al. [1] - and, according to a general argument, their mass at formation, $M_{B H}\left(t_{f}\right)$, is proportional to the formation time, $t_{f}$,

$$
\frac{t_{f}}{\hbar}=\frac{1}{\gamma} \frac{M_{B H}\left(t_{f}\right) c^{2}}{\left(M_{P l} c^{2}\right)^{2}}
$$

where $\hbar$ is the reduced Planck constant, $\gamma$ is a numerical factor (often taken to be equal to 0.2), $M_{P l} \simeq 2.2 \times 10^{-5} \mathrm{~g}$ is the Planck mass and $c$ is the speed of light in vacuum.

Here we consider PBHs that, at the formation time, were heavier than $M_{P l}$, but lighter than about $10^{9} \mathrm{~g}$, in which case the evaporation happened before Big Bang Nucleosynthesis (BBN). It is useful to introduce the parameter $\beta$, defined as the PBHs over the radiation mass density at the formation time, $\beta=\rho_{B H}\left(t_{f}\right) / \rho_{R}\left(t_{f}\right)$. Depending on $\beta$, the Universe might have been radiation or BH dominated at the evanescence of the PBHs [2]: these situations are referred to as radiation or $\mathrm{BH}$ domination, respectively. The range of PBHs masses between $10^{-5} \mathrm{~g}$ and $10^{9} \mathrm{~g}$ is quite unconstrained, apart from gravitational waves (GW) induced by second order effects [3, 4], which set an upper limit on $\beta$, excluding a small portion of the region associated to $\mathrm{BH}$ domination.

Let us review the evaporation mechanism [5-8] for a generic rotating Kerr PBH, characterized by the adimensional spin parameter $a_{*}=(J / \hbar) M_{P l}^{2} / M_{B H}^{2}$, where $J$ is the PBH angular momentum. The non-rotating Schwarzschild case is recovered in the limit $a_{*}=0$. The evaporation process is such that, at time $t$, all particles are emitted whose mass is smaller than the Hawking temperature

$$
k_{B} T_{B H}(t)=\frac{1}{8 \pi} \frac{\left(M_{P l} c^{2}\right)^{2}}{M_{B H} c^{2}} \frac{2}{1+\frac{1}{\sqrt{1-a_{*}(t)^{2}}}},
$$

where $k_{B}$ is the Boltzmann constant. For each particle of type $i$, with spin $s_{i}$ and mass $m_{i}$, emitted by a single $\mathrm{PBH}$, the instantaneous energy distribution per particle degree of freedom (dof) is given by (here we follow the conventions of [9])

$$
\frac{1}{g_{i}} \frac{d^{2} N_{i}}{d t d E}=\frac{1}{2 \pi \hbar} \sum_{\ell, m} \Gamma_{s_{i} \ell m}\left(E, M_{B H}, a_{*}(t)\right) \frac{1}{e^{\frac{E^{\prime}}{k_{B} T_{B H}(t)}}-(-1)^{2 s_{i}}},
$$

where $E^{\prime}=E-m \Omega$ is the total energy of the particles, taking into account the BH horizon rotation velocity on top of the total energy $E^{2}=(c p)^{2}+\left(m_{i} c^{2}\right)^{2}, m$ is the particle angular momentum projection, the greybody factors $\Gamma$ are dimensionless absorption probabilities for the emitted species and $g_{i}$ are the internal dof of the $i$-th particle, which account for the polarization and color dof. In the Schwarzschild case, the high energy $(E \rightarrow \infty)$ behavior is well reproduced by the geometrical optics approximation

$$
\Gamma_{s_{i}} \rightarrow \frac{27}{(8 \pi)^{2}}\left(\frac{E}{k_{B} T_{B H}(t)}\right)^{2}
$$

The public code BlackHawk $[9,10]$ numerically computes the greybody factors, enabling one to obtain the instantaneous spectrum for each type of emitted particle with spin $s_{i}=0,1 / 2,1,3 / 2,2$. 
The peak of the energy distribution is at $E \approx k_{B} T_{B H}(t)$. If the particle is sufficiently light, the ultra relativistic limit applies, $E \approx c p(t)$.

Let us define the ratio $f(t)=\rho_{B H}(t) / \rho_{R}(t)$. Denoting the scale factor with $a(t)$, since $\rho_{B H}(t) \propto 1 / a(t)^{3}$, while $\rho_{R}(t) \propto 1 / a(t)^{4}$, the ratio $f(t)$ increases linearly in the scale factor, $f(t) \propto a(t)$. It is possible that PBHs dominate the energy content of the Universe before their complete evaporation: this a situation is referred to as $\mathrm{BH}$ domination. The case in which complete evaporation takes place before $\mathrm{PBHs}$ domination might occur, is referred to as radiation domination. We define $\bar{\beta}$ to be the maximum value of $\beta$ corresponding to radiation domination, that is the value of $\beta$ leading to $f\left(t_{e v}\right)=1$. One finds that $\bar{\beta}=\frac{f\left(t_{f}\right)}{f\left(t_{e v}\right)}=\frac{a\left(t_{f}\right)}{a\left(t_{e v}\right)}$. For $\beta \lesssim \bar{\beta}$, radiation domination occurs, while for $\beta \gtrsim \bar{\beta}$, BH domination occurs.

The distribution of the momentum at evaporation for the particle of type $i$, normalized per dof, is a superposition of all the instantaneous distributions, each redshifted appropriately from its time of emission $t_{e m}$ to the evaporation time $t_{e v}$

$$
\frac{1}{g_{i}} \frac{d N_{i}}{d(c p)}\left(t_{e v}\right)=\int_{t_{e m}}^{t_{e v}} d t \frac{d^{2} N}{d t d(c p(t))}(\underbrace{c p\left(t_{e v}\right) \frac{a\left(t_{e v}\right)}{a(t)}}_{c p(t)}, T_{B H}(t), a_{*}(t)) \frac{a\left(t_{e v}\right)}{a(t)},
$$

and we refer to refs. [11, 12] fort more details. In order to get rid of the PBH mass dependence, it is useful to define the adimensional momentum $x\left(t_{e v}\right)=c p\left(t_{e v}\right) /\left(k_{B} T_{B H}^{S}\right)$, where $T_{B H}^{S}=\left.T_{B H}\left(t_{f}\right)\right|_{a_{*}=0}$, with the related adimensional momentum distribution at evaporation

$$
\tilde{F}\left(x\left(t_{e v}\right)\right) \equiv \frac{\left(k_{B} T_{B H}^{S}\right)^{3}}{\left(M_{P l} c^{2}\right)^{2}} \frac{1}{g_{i}} \frac{d N_{i}}{d(c p)}\left(t_{e v}\right),
$$

which has the property of depending only on the particle spin $s_{i}$ and on the BH spin parameter, $a_{*}$, while being independent of the PBH mass.

For the Schwarzschild case [11], the quantity $\tilde{F}\left(x\left(t_{e v}\right)\right)$ derived from BlackHawk [9] is shown in the left plot of fig. 1, assuming radiation domination with $\beta=\bar{\beta}$ (solid) and full (namely a long period of) BH domination (dotted). The suppression due to the different values of the emitted particle spin is manifest. The right plot shows the same for the Kerr case [12], taking $a_{*}=0.8$. The contribution at small $x\left(t_{e v}\right)$ is particularly sensitive to the rotating regime: the higher is the value of PBH spin parameter $a_{*}$, the more the contribution from particles with high spin, like $s=2$ and $s=1$, gets enhanced.

\section{Stable particles from evaporating PBHs as DM}

It has been proposed that particles beyond the SM, possibly produced via the PBHs evaporation mechanism, might be responsible for the excess of baryons over anti-baryons [5], that they might account for some or all of the DM observed today [2, 11-16] and that they might provide a contribution to Dark Radiation (DR) [12-14, 16, 17]. Here we review the status of the possibility that a stable beyond the SM particle emitted in the PBHs evaporation mechanism fully accounts for the DM observed today, summarizing some of the results of our works, refs. [11, 12, 14]. 

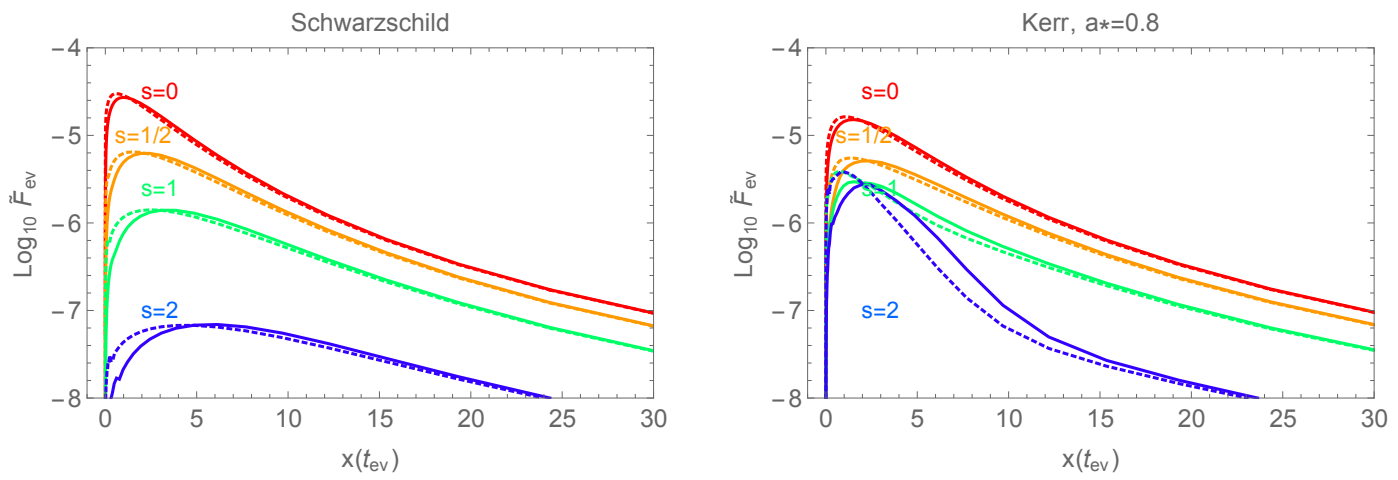

Figure 1: Adimensional function $\tilde{F}\left(x\left(t_{e v}\right)\right)$, as defined by eq. (6), assuming radiation domination with $\beta=\bar{\beta}$ (solid) or full BH domination (dashed), calculated using BlackHawk [9] within the SM and for various particle spins, as indicated. Left: Schwarzschild case. Right: Kerr case with $a_{*}=0.8$.

We denote by $X$ such a stable particle. It turns out that, in the Schwarzschild case, there are two possibilities for the DM mass, which are denoted as "light" and "heavy" DM [2], according to the fact that the $X$ particles are emitted respectively during all the PBH lifetime or just in its final stages. If $m_{X} c^{2}<k_{B} T_{B H}^{S}, \mathrm{BH}$, the DM candidate belongs to the light category, otherwise to the heavy one.

As reference DM case, we consider emission of a scalar boson (with $g_{X}=1$ ) from a Schwarzschild PBH. The associated value of the DM mass giving the full contribution to DM is denoted by $\bar{m}$. We display the iso-contours of $\log _{10}\left(\bar{m} c^{2}[\mathrm{GeV}]\right)$ in the left and right plots of fig. 2, for the light and heavy case respectively [14]. The diagonal line separating the two regimes of $\mathrm{BH}$ domination (upper right) and radiation domination (lower left) corresponds to $\beta=\bar{\beta}$.
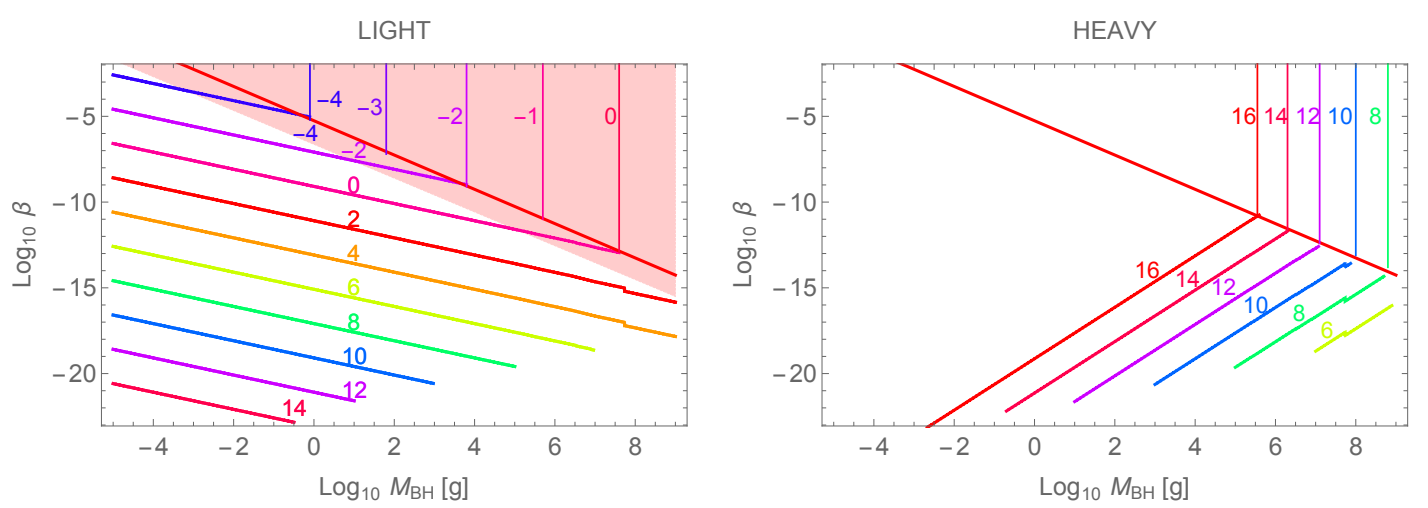

Figure 2: Iso-contours of $\log _{10}\left(\bar{m} c^{2}[\mathrm{GeV}]\right)$ for which a scalar $X$ particle $\left(s_{X}=0\right)$ accounts for the observed amount of DM today, for the "light" and "heavy" case respectively [14].

The light DM scenario is particularly interesting because the range of DM masses goes from $10^{-4} \mathrm{GeV}$ to about $1 \mathrm{GeV}$. In the heavy DM scenario, the range of DM masses goes instead from $10^{8}$ $\mathrm{GeV}$ to about $10^{16} \mathrm{GeV}$; it might be more difficult from the model building point of view to have a 
stable particle with such a high mass value. The light DM case has however to face a problem with structure formation $[2,11,13-15]$. The spectrum of the DM candidate is indeed quite hard, as the DM particles are emitted with a momentum of the order of the Hawking temperature of the PBH, so that they turn out to be warm DM candidates, able to have a negative impact on structure formation. The shaded area in the left plot of fig. 2, which fully includes the region of $\mathrm{BH}$ domination $(\beta>\bar{\beta})$, turns out to be excluded because of the constraints from structure formation (see [11, 14] for more details).

It is worth to investigate whether different values of the $X$ particle spin, such as $s_{X}=$ $1 / 2,1,3 / 2,2$, might reduce the tension with structure formation, thus allowing some portion of the $\mathrm{BH}$ domination region. The generalization to other values of the $X$ particle spin for the Schwarzschild case is discussed in ref. [11]: the result is that the tension is indeed reduced as $s_{X}$ is increased from 0 up to 2, but this is insufficient to "save" the BH domination region. It is also worth to investigate the effect of an initial spin for the PBHs, as one might guess that the generalization to Kerr PBHs might be successful in alleviating the tension with structure formation. The result of the detailed analysis [12] is that: for $s_{X}=0,1 / 2,1$, there is no significant difference with respect to the Schwarzschild case, as for the impact on structure formation; while for $s_{X}=2$, the tension with structure formation is even exacerbated with respect to the Schwarzschild case.

\section{Conclusions}

Evaporation of PBHs with masses between $10^{-5} \mathrm{~g}$ and $10^{9} \mathrm{~g}$ is an elegant and viable mechanism to account for the DM observed today. In the heavy DM case, both radiation and $\mathrm{BH}$ domination regions are allowed. In the light DM case, only the radiation domination region is allowed, due to constraints from structure formation; for the $\mathrm{BH}$ domination region, the tension with structure formation is not avoided by invoking a high spin for the DM particles [11], nor by allowing the PBHs to be rotating [12]. Successful ways to avoid the tension with structure formation for the light DM case with BH domination have been proposed, like e.g. entropy production [2] and thermalization of self-interacting DM particles with number changing interactions [18].

\section{References}

[1] B. Carr, K. Kohri, Y. Sendouda and J. Yokoyama, Constraints on Primordial Black Holes, [arXiv:2002.12778 [astro-ph.CO]].

[2] T. Fujita, M. Kawasaki, K. Harigaya and R. Matsuda, Baryon asymmetry, dark matter, and density perturbation from primordial black holes, Phys. Rev. D 89 (2014) no.10, 103501 doi:10.1103/PhysRevD.89.103501 [arXiv:1401.1909 [astro-ph.CO]].

[3] T. Papanikolaou, V. Vennin and D. Langlois, Gravitational waves from a universe filled with primordial black holes, JCAP 03 (2021), 053 doi:10.1088/1475-7516/2021/03/053 [arXiv:2010.11573 [astro-ph.CO]].

[4] G. Domènech, C. Lin and M. Sasaki, Gravitational wave constraints on the primordial black hole dominated early universe, JCAP 04 (2021), 062 doi:10.1088/1475-7516/2021/04/062 [arXiv:2012.08151 [gr-qc]]. 
[5] S. W. Hawking, Black hole explosions, Nature 248 (1974), 30-31 doi:10.1038/248030a0

[6] S. W. Hawking, Particle Creation by Black Holes, Commun. Math. Phys. 43 (1975), 199-220 [erratum: Commun. Math. Phys. 46 (1976), 206] doi:10.1007/BF02345020

[7] D. N. Page, Particle Emission Rates from a Black Hole: Massless Particles from an Uncharged, Nonrotating Hole, Phys. Rev. D 13 (1976), 198-206 doi:10.1103/PhysRevD.13.198

[8] D. N. Page, Particle Emission Rates from a Black Hole. 2. Massless Particles from a Rotating Hole, Phys. Rev. D 14 (1976), 3260-3273 doi:10.1103/PhysRevD.14.3260

[9] A. Arbey and J. Auffinger, BlackHawk: A public code for calculating the Hawking evaporation spectra of any black hole distribution, Eur. Phys. J. C 79 (2019) no.8, 693 doi:10.1140/epjc/s10052-019-7161-1 [arXiv:1905.04268 [gr-qc]].

[10] A. Arbey and J. Auffinger, Physics Beyond the Standard Model with BlackHawk v2.0, [arXiv:2108.02737 [gr-qc]].

[11] J. Auffinger, I. Masina and G. Orlando, Bounds on warm dark matter from Schwarzschild primordial black holes, Eur. Phys. J. Plus 136 (2021) no.2, 261 doi:10.1140/epjp/s13360-02101247-9 [arXiv:2012.09867 [hep-ph]].

[12] I. Masina, Dark matter and dark radiation from evaporating Kerr primordial black holes, [arXiv:2103.13825 [gr-qc]]. To appear on Gravitation and Cosmology 27 (2021) no.4, 315.

[13] O. Lennon, J. March-Russell, R. Petrossian-Byrne and H. Tillim, Black Hole Genesis of Dark Matter, JCAP 04 (2018), 009 doi:10.1088/1475-7516/2018/04/009 [arXiv:1712.07664 [hep-ph]].

[14] I. Masina, Dark matter and dark radiation from evaporating primordial black holes, Eur. Phys. J. Plus 135 (2020) no.7, 552 doi:10.1140/epjp/s13360-020-00564-9 [arXiv:2004.04740 [hep-ph]].

[15] I. Baldes, Q. Decant, D. C. Hooper and L. Lopez-Honorez, Non-Cold Dark Matter from Primordial Black Hole Evaporation, JCAP 08 (2020), 045 doi:10.1088/1475-7516/2020/08/045 [arXiv:2004.14773 [astro-ph.CO]].

[16] D. Hooper, G. Krnjaic and S. D. McDermott, Dark Radiation and Superheavy Dark Matter from Black Hole Domination, JHEP 08 (2019), 001 doi:10.1007/JHEP08(2019)001 [arXiv:1905.01301 [hep-ph]].

[17] D. Hooper, G. Krnjaic, J. March-Russell, S. D. McDermott and R. Petrossian-Byrne, Hot Gravitons and Gravitational Waves From Kerr Black Holes in the Early Universe, [arXiv:2004.00618 [astro-ph.CO]].

[18] N. Bernal and Ó. Zapata, Self-interacting Dark Matter from Primordial Black Holes, JCAP 03 (2021), 007 doi:10.1088/1475-7516/2021/03/007 [arXiv:2010.09725 [hep-ph]]. 